\title{
Burkholderia cepacia complex bacteremia outbreaks among non-cystic fibrosis patients in the pediatric unit of a university hospital
}

\author{
Sinan Tüfekci ${ }^{1 \oplus}$, Birol Şafak ${ }^{2 \oplus}$, Burçin Nalbantoğlu ${ }^{1 \oplus}$, Nedim Samanci ${ }^{1 \oplus}$, \\ Nuri Kiraz ${ }^{2 \oplus}$ \\ Departments of ${ }^{1}$ Pediatrics and ${ }^{2}$ Microbiology, Namık Kemal University Faculty of Medicine, Tekirda $\breve{g}$, Turkey.
}

\begin{abstract}
Background. Burkholderia cepacia complex (Bcc) comprises multi-drug resistant, Gram-negative, motile, and aerobic bacteria. Bcc causes severe nosocomial infections particularly in patients with intravascular catheters and in those with cystic fibrosis. We studied a Bcc outbreak in non-cystic fibrosis patients.

Methods. We analyzed data from six patients hospitalized at our center. Blood cultures identified as infectious were incubated onto $5 \%$ blood sheep agar, chocolate agar, and eosin methylene blue (EMB) agar. We examined possible sites that could be sources of infection at the clinic. We confirmed isolations with pulsed-field gel electrophoresis (PFGE) tests.

Results. The first patient was hospitalized due to left renal agenesis, urinary tract infection, and renal failure. Bcc was isolated in blood cultures obtained due to high fever on the third day of hospitalization. We stopped new patient hospitalizations after detecting Bcc in blood cultures of other five patients. We did not detect further positive specimens obtained from other clinic and the patient rooms. PFGE patterns were similar in all clinical isolates of Bcc indicating that the outbreak had originated from the source.

Conclusions. Bcc infection should be considered in cases of nosocomial outbreaks of multi-drug resistant organisms that require hospitalization at intensive care units. Control measures should be taken for prevention of nosocomial infections and required investigations should be done to detect the source of infection.
\end{abstract}

Key words: Burkholderia cepacia complex, child, outbreak, infection control, non-cystic fibrosis.

Burkholderia cepacia complex (Bcc) are aerobic, oxidase positive, motile, non-fermentative, spore-free Gram-negative bacilli that can cause opportunistic infections. They are commonly found in soil and humid environments. Bcc group includes at least 21 species that are phenotypically similar, but genotypically different. Identifying them is difficult with routine biochemical tests, and it may yield incorrect results. Therefore, confirmation and molecular tests should be performed in reference laboratories. ${ }^{1}$ Bcc have emerged as pathogens

Sinan Tüfekci

stufekci@nku.edu.tr

Received 14th November 2019, revised 20th April 2020, accepted 11st May 2020. leading to necrotizing pneumonia and bacteremia that is intrinsically resistant to most antibiotics, particularly in patients with cystic fibrosis and chronic granulomatous disease. ${ }^{2,3}$ However, their pathogenicity is not limited to these patients. The bacteria may colonize and infect the respiratory tract, blood stream, and urinary tract in immunocompromised patients. Bcc infections have been reported in intensive care units, in patients on dialysis, transplant patients, newborns, and those with intravenous catheters. Majority of Bcc isolates are intrinsically resistant to aminoglycosides, polymyxins, and other beta-lactam antibiotics. These bacteria are highly contagious in hospital environments and early diagnosis and treatment of patients is essential. 
We investigated a bacteremia outbreak in six patients, who were detected to have Bcc bacilli growing in blood cultures and hospitalized in our pediatric clinic.

\section{Material and Methods}

We analyzed data from six patients hospitalized at our pediatric clinic between 18 and 25 February 2019, who had Bcc growing in blood cultures. The ethics committee of Tekirdağ Namık Kemal University Faculty of Medicine approved the study (date and report number: 2019-83.06.04).Written informed consent was received from all.

Epidemiologic research: We initiated our study rapidly after discussing the detection of Bccpositive blood stream infections in six patients of our clinic with the infection control committee. We obtained clinical records of the patients. The staff of the clinic was informed about blood culture obtaining techniques. Compliance to infection control measures was checked. The training of health care workers, hand hygiene, use of gloves and skin antisepsis during blood culture, how environmental cleaning is done, disinfection and sterilization applications, surveillance studies were checked.

We obtained cultures from potential infection sources including intravenous fluid administration, vial stopper, disinfectants, antiseptics, nebulizer solutions, drugs, syringes, environmental surface, other patients, personnel, cotton balls, gauze, blood culture bottles, and distilled water. Humidified sterile swabs were used. Blood was directly cultivated in $5 \%$ sheep blood, eosin methylene blue (EMB), and chocolate agars and incubated at $36 \pm 1{ }^{\circ} \mathrm{C}$ for 48 hours. Fluid samples were additionally inoculated into automated blood culture vials and left for five days of incubation.

Microbiological analysis: The BACTEC 9120 (Becton Dickinson, USA) device was used for bacterial detections. Blood culture samples which yielded positive signals were cultivated onto 5\% sheep blood agar and EMB agar and incubated at $36 \pm 1^{\circ} \mathrm{C}$ for $24-48$ hours. We used a Vitek 2 system (Biomerieux, Marcy L'etoile, France) and conventional methods for biochemical identification and antibiotic susceptibility testing.

Confirmation of isolates and PFGE tests were performed at the Ministry of Health, General Directorate of Public Health Presidency of Microbiology Reference Laboratories and Biological Products Department. Deoxyribonucleic acid (DNA) extractions were made from the colonies in the growth media. Afterwards we digested DNA samples with the restriction enzyme Fast Digest Spel (Thermo Scientific, USA). We performed a fingerprint assay using PFGE to investigate the clonal identity of the clinical isolates. Molecular weight standards were used as a control for PFGE experiment.

\section{Results}

Of the six patients detected to have Bcc growing in their blood cultures, four were female and two were male. Their ages ranged from 8 months to 14.5 years. B. cepacia was isolated from blood cultures of 6 patients between 18-25 February, 2019 (Table I).

The patients did not have a history of congenital anomaly, growth or developmental retardation, chronic diarrhea, immune deficiency, or cystic fibrosis. Their weights and heights were normal for their age. The first patient was hospitalized due to left renal agenesis, urinary tract infection, and renal failure. Bcc growth was detected in blood cultures obtained due to high fever on the third day after hospital admission. We stopped admitting new patients to the clinic after detecting Bcc growth in the blood cultures of the other five patients in the clinic. The third and fourth patients required mechanical ventilation at the intensive care unit after they developed respiratory failure.

The bacteria in the blood cultures of all patients were identified as Bcc. All strains were susceptible to trimethoprim/sulfamethoxazole 
Table I. Clinical features of the cases.

\begin{tabular}{|c|c|c|c|c|c|c|}
\hline Cases & Gender & Age & First diagnosis & $\begin{array}{c}\text { Date for positive blood } \\
\text { culture for B. cepacia }\end{array}$ & $\begin{array}{l}\text { Ventilator } \\
\text { care }\end{array}$ & $\begin{array}{c}\text { Duration of hospital } \\
\text { stay (days) }\end{array}$ \\
\hline \multirow[t]{5}{*}{1} & \multirow[t]{5}{*}{ Female } & \multirow[t]{5}{*}{14.5 years } & \multirow{5}{*}{$\begin{array}{l}\text { Left renal agenesis, } \\
\text { urinary infection }\end{array}$} & $18.02 .2019(+)$ & \multirow[t]{5}{*}{ No } & \multirow[t]{5}{*}{12} \\
\hline & & & & $20.02 .2019(+)$ & & \\
\hline & & & & 22.02.2019 (+) & & \\
\hline & & & & $24.02 .2019(+)$ & & \\
\hline & & & & 27.02.2019 (-) & & \\
\hline \multirow[t]{2}{*}{2} & \multirow[t]{2}{*}{ Male } & \multirow[t]{2}{*}{14.3 years } & \multirow[t]{2}{*}{ Vasculitis } & 20.02.2019 (+) & \multirow[t]{2}{*}{ No } & \multirow[t]{2}{*}{7} \\
\hline & & & & 27.02.2019 (-) & & \\
\hline \multirow[t]{3}{*}{3} & \multirow[t]{3}{*}{ Male } & \multirow[t]{3}{*}{20 months } & \multirow[t]{3}{*}{ Pneumonia } & $22.02 .2019(+)$ & \multirow[t]{3}{*}{ Yes } & \multirow[t]{3}{*}{21} \\
\hline & & & & 02.03.2019 (+) & & \\
\hline & & & & 08.03.2019 (-) & & \\
\hline \multirow[t]{2}{*}{4} & \multirow[t]{2}{*}{ Female } & \multirow[t]{2}{*}{8 months } & \multirow{2}{*}{$\begin{array}{l}\text { Pneumonia and } \\
\text { urosepsis }\end{array}$} & 23.02.2019 (+) & \multirow[t]{2}{*}{ Yes } & \multirow[t]{2}{*}{10} \\
\hline & & & & 28.02.2019(-) & & \\
\hline \multirow[t]{2}{*}{5} & \multirow[t]{2}{*}{ Female } & \multirow[t]{2}{*}{4.7 years } & \multirow[t]{2}{*}{ Pneumonia } & 24.02.2019 (+) & \multirow[t]{2}{*}{ No } & \multirow[t]{2}{*}{7} \\
\hline & & & & 28.02.2019(-) & & \\
\hline \multirow[t]{3}{*}{6} & \multirow[t]{3}{*}{ Female } & \multirow[t]{3}{*}{3.5 years } & \multirow[t]{3}{*}{ Acute bronchiolitis } & $25.02 .2019(+)$ & \multirow[t]{3}{*}{ No } & \multirow[t]{3}{*}{14} \\
\hline & & & & 28.02.2019 (+) & & \\
\hline & & & & 03.03.2019 (-) & & \\
\hline
\end{tabular}

and resistant to ceftazidime (minimum inhibitory concentration, MIC: $16 \mathrm{mg} / \mathrm{L}$ ), and intermediately susceptible to meropenem (MIC: $4 \mathrm{mg} / \mathrm{L})$.

Molecular typing of PFGE confirmed the clonal identical between the cepacia isolates from the blood of the 6 patients. PFGE patterns were similar in all clinical isolates indicating that the outbreak originated from a single source (Fig. 1). We did not detect growth from samples obtained from the clinic and patient rooms. We were not able to identify the primary source. The

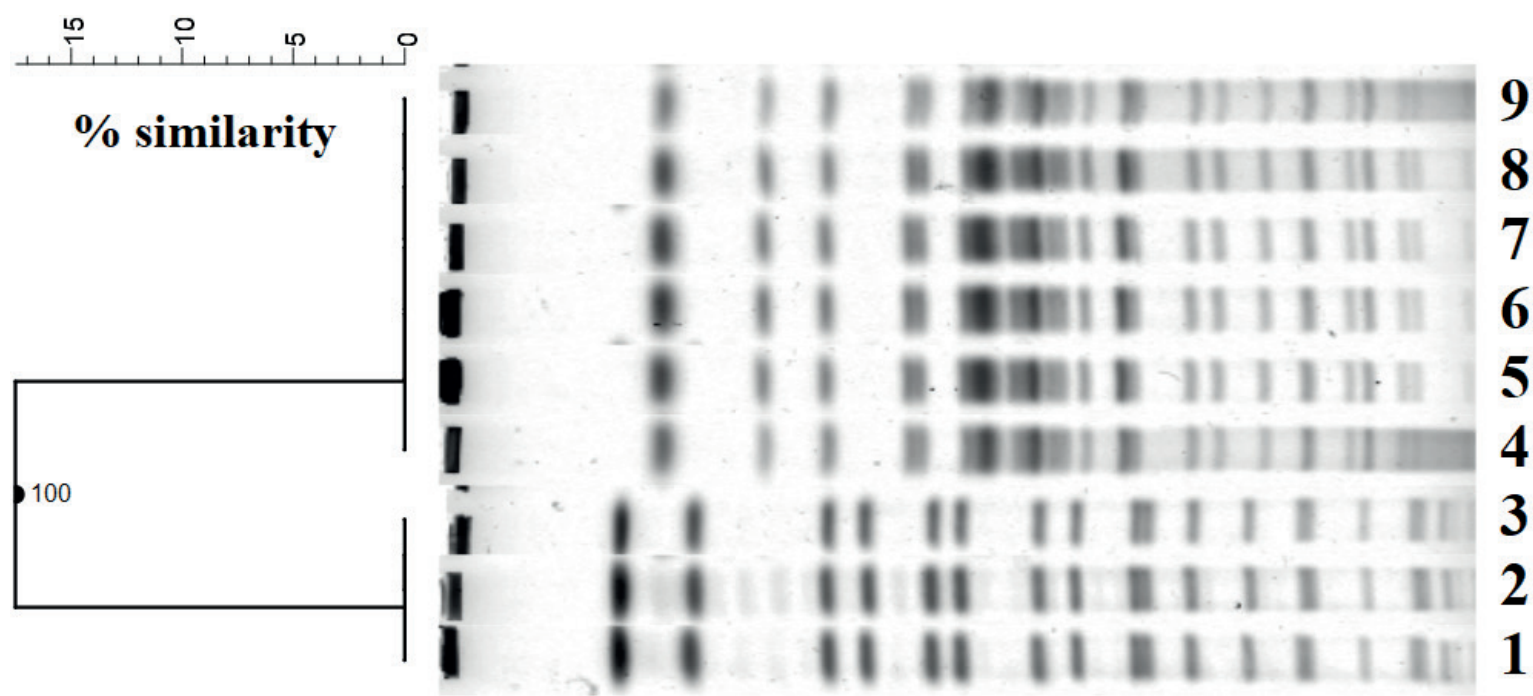

Fig. 1. Pulsed-field gel electrophoresis patterns for 6 clinical isolates. Lane 1-3, molecular weight standards; Lane 4-9, clinical isolates. 
infected patients recovered after trimethoprim/ sulfamethoxazole treatment within 7 to 21 days and were discharged (Table I). No growths were detected in control blood cultures after treatment.

\section{Discussion}

B. cepacia complex has recently been added to the group of non-fermentative Gram-negative bacteria including Pseudomonas aeruginosa and Acinetobacter baumannii. ${ }^{4} \mathrm{Bcc}$ is found in water sources, soil, plants, and nature. It can lead to outbreaks through different sources such as contaminated taps, sinks, various intravenous and irrigation solutions, saline solutions, nebulizer drugs, respiratory devices using tap or distilled water, catheters, dialysis fluids and machines, blood gas measurement devices, thermometers, ventilator heat sensors, containers for enteral feeding, disinfectants (chlorhexidine), and antiseptics including povidone iodine, intravenous caffeine citrate, ultrasound gel, moisturizer, and benzalkonium chloride. ${ }^{5-10}$ The source was detected in 22 of 30 Bcc outbreaks reported in non-cystic fibrosis patients in intensive care units. ${ }^{111,12}$ However, the source in the remaining eight outbreaks could not be detected. We also failed to detect a source in the outbreak at our pediatric clinic despite detailed investigations done following reference literature recommendations.

The presence of a central venous catheter, hemodialysis-requiring renal failure, the requirements for multiple bronchoscopies, and recent surgeries have been reported as risk factors for Bcc bacteremia in case-control studies. The need for and duration of mechanical ventilator and the need for tracheostomy have been reported to increase the risk of acquiring Bcc infections compared to control groups. ${ }^{13}$ Our patients did not have a history of central venous catheterization, invasive interventions, hemodialysis, surgery or bronchoscopy. The hospital stay was three weeks in the third patient as mechanical ventilation was indicated during treatment.
Bcc may spread from one person to another directly through infected excretions and droplets, or indirectly through contaminated devices and equipment. Isolation of the infected patient is of great importance. As a result of the outbreak at our hospital, we checked sterilization conditions, measures for isolation, hand hygiene, use of disposable gloves and masks. We shortened duration of visits, and improved health professional's education and environmental factors.

Bcc rarely leads to infections in healthy individuals and has a low mortality and morbidity despite having a high intrinsic resistance to many antimicrobial and antiseptic agents. ${ }^{14,15}$ Bcc may lead to life-threatening opportunistic infections like urinary tract infection, septic arthritis, peritonitis, bacteremia, sepsis, osteomyelitis, meningitis, pulmonary abscess, and pneumonia in susceptible patients, particularly in patients in intensive care units and those with underlying diseases such chronic granulomatous disease, oncologic conditions, cystic fibrosis, or other immunocompromised patients, who are continually applied catheters/ medical devices. Secondary urogenital infections may also be due to urogenital interventions. ${ }^{6,16}$ The rates of intensive care unit hospitalizations were reported to be $61.9 \%$ and $52.9 \%$ by Dizbay and by Srinivasan, respectively . This rate was $33 \%$ in our patients. The mortality rate has been reported to be $41-83 \%$ in Bcc-related infections. ${ }^{3,4}$

While the vast majority of Bcc outbreaks originate in intensive care units, our patients were hospitalized at the pediatric clinic. We suspected that a 10-month old infant discharged after completion of her 10-day treatment for cystic fibrosis and recurrent pneumonia was the original outbreak source, but we did not detect bacterial growth in her samples. Our first Bcc $(+)$ patient stayed in that room from which that cystic fibrosis case was discharged one week prior. There might be a connection between these two cases. 
Bcc are among the nosocomial opportunistic microorganisms causing outbreaks in intensive care units due to their natural resistance to many antibiotics. They are associated with high mortality and morbidity in newborns, and in pediatric and adult intensive care units. Removing the main source, isolating patients, keeping hand hygiene, using disposable gloves and masks, keeping visits short, educating the staff, and cleaning and disinfecting the environment are important factors to prevent the spread of outbreaks. In our study, outbreaks were terminated in a short time through infection control measures taken rapidly after detecting Bcc bacteremia in pediatric clinics, even though their source could not be detected. Bcc infection should be considered in children hospitalized in general and intensive care units and in whom non-specific antibiotic treatment response is unsatisfactory.

\section{REFERENCES}

1. Abdallah M, Abdallah HA, Memish ZA. Burkholderia cepacia complex outbreaks among non-cystic fibrosis patients in the intensive care units: a review of adult and pediatric literature. Infez Med 2018; 26: 299-307.

2. Mali S, Dash L, Gautam V, Shastri J, Kumar S. An outbreak of Burkholderia cepacia complex in the paediatric unit of a tertiary care hospital. Indian J Med Microbiol 2017; 35: 216-220.

3. Dizbay M, Tunccan OG, Sezer BE, Aktas F, Arman D. Nosocomial Burkholderia cepacia infections in a Turkish university hospital: a five-year surveillance. J Infect Dev Ctries 2009; 3: 273-277.

4. Srinivasan S, Arora NC, Sahai K. Report on the newly emerging nosocomial Burkholderia cepacia in a tertiary hospital. Med J Armed Forces India 2016; 72 (Suppl 1): S50-S53.

5. Mahenthiralingam E, Baldwin A, Dowson CG. Burkholderia cepacia complex bacteria: opportunistic pathogens with important natural biology. J Appl Microbiol 2008; 104: 1539-1551.

6. Leitao JH, Sousa SA, Ferreira AS, Ramos CG, Silva IN, Moreira LM. Pathogenicity, virulence factors, and strategies to fight against Burkholderia cepacia complex pathogens and related species. Appl Microbiol Biotechnol 2010; 87: 31-40.
7. Antony B, Cherian EV, Boloor R, Shenoy KV. A sporadic outbreak of Burkholderia cepacia complex bacteremia in pediatric intensive care unit of a tertiary care hospital in coastal Karnataka, South India. Indian J Pathol Microbiol 2016; 59: 197-199.

8. Ko S, An HS, Bang JH, Park SW. An outbreak of Burkholderia cepacia complex pesudobacteremia associated with intrinsically contaminated commercial $0.5 \%$ chlorhexidine solution. Am J Infect Control 2015; 43: 266-268.

9. Song JE, Kwak YG, Um TH, et al. Outbreak of Burkholderia cepacia pseudobacteraemia caused by intrinsically contaminated commercial $0.5 \%$ chlorhexidine solution in neonatal intensive care units. J Hosp Infect 2018; 98: 295-298.

10. Heo ST, Kim SJ, Jeong YG, Bae IG, Jin JS, Lee JC. Hospital outbreak of Burkholderia stabilis bacteraemia related to contaminated chlorhexidine in haematological malignancy patients with indwelling catheters. J Hosp Infect 2008; 70: 241-245.

11. Hamill RJ, Houston ED, Georghiou PR, et al. An outbreak of Burkholderia (formerly Pseudomonas) cepacia respiratory tract colonization and infection associated with nebulized albuterol therapy. Ann Intern Med 1995; 122: 762-766.

12. Righi E, Girardis M, Marchegiano $P$, et al. Characteristics and outcome predictors of patients involved in an outbreak of Burkholderia cepacia complex. J Hosp Infect 2013; 85: 73-75.

13. Bressler AM, Kaye KS, LiPuma JJ, et al. Risk factors for Burkholderia cepacia complex bacteremia among intensive care unit patients without cystic fibrosis: a case-control study. Infect Control Hosp Epidemiol 2007; 28: 951-958.

14. Martin M, Christiansen B, Caspari G, et al. Hospitalwide outbreak of Burkholderia contaminans caused by prefabricated moist washcloths. J Hosp Infect 2011; 77: 267-270.

15. Shrivastava B, Sriram A, Shetty S, Doshi R, Varior R. An unusual source of Burkholderia cepacia outbreak in a neonatal intensive care unit. J Hosp Infect 2016; 94: 358-360.

16. LiPuma JJ, Currie BJ, Lum GD, Vandamme PAR. Burkholderia, stenotrophomonas, ralstonia, cupriavidus, pandoraea, brevundimonas, comamonas, delftia, and acidovorax. In: Murray PR, Baron EJ, Jorgensen JH, Landry ML, Pfaller MA (eds). Manual of Clinical Microbiology.Washington DC: ASM Press, 2007: 749-769. 